\title{
IMMUNODIAGNOSIS OF ANGIOSTRONGYLIASIS WITH MONOCLONAL ANTIBODIES RECOGNIZING A CIRCULATING ANTIGEN OF MOL.WT 91,000 FROM ANGIOSTRONGYLUS CANTONENSIS
}

\author{
H. H. SHIH and S. N. CHEN \\ Department of Zoology, National Taiwan University, Taipei, Taiwan, Republic of China
}

(Received 29 March 1990; accepted 23 October 1990)

\begin{abstract}
SHIH H. H. and CHEN S. N. 1991. Immunodiagnosis of angiostrongyliasis with monoclonal antibodies recognizing a circulating antigen of mol.wt 91,000 from Angiostrongylus cantonensis. International Journal for Parasitology 21: 171-177. In the analysis of excretory-secretory (ES) antigens from infective third-stage larvae (L3) of Angiostrongylus cantonensis, one major component of mol.wt 91,000 was not precipitated by pooled sera of patients with eosinophilic meningoencephalitis. Monoclonal antibodies $(\mathrm{Mc} A \mathrm{~b})$ secreted from two hybridoma cell lines, established against somatic antigens of $L 3$, recognized this molecule but with different epitope specificities indicated by an additivity index (A.I.) of $83 \%$. The $2 \mathrm{Mc} \mathrm{Ab}$ (TD2 and 3A5) belonged to IgG2a and IgM classes, respectively. Combinations of TD2 and 3A5 were used in a sensitive enzyme-linked fluorescent assay (ELFA) for the immunodiagnosis of human angiostrongyliasis. The double-antibody sandwich ELFA method was applied firstiy to sera from experimentally infected rats using either TD2 or 3A5 to coat the assay plates. Two tluorescence unit (F.U.) peaks appeared in sera from infected rats collected 18 and 44 days after infection. Specimens from 35 patients were tested, all cerebrospinal fluids (CSF) and most sera ( $88 \%$ ) showed positive reactions and the average F.U. of CSF was greater than that of serum.
\end{abstract}

INDEX KEY WORDS: Angiostrongylus cantonensis; monoclonal antibody; enzyme-linked fluorescence assay; immunodiagnosis.

\section{INTRODUCTION}

Angiostrongylus cantonensis causes eosinophilic meningoencephalitis in humans and is found commonly in Southeast Asia and the Pacific Islands (Alicata \& Jindrak, 1970; Cross, 1978). Hundreds of human cases were recorded over the last decade in Taiwan. Definite diagnosis of angiostrongyliasis relies on the discovery of either larval or juvenile worms in the CSF of patients. Such diagnoses are difficult to make because worms are seldom found in the limited volume of CSF. recovered. Immunodiagnosis can be useful in such circumstances. The indirect enzyme-linked immunosorbent assay (ELISA) is preferred for detecting the presence and titre of antibody $(A b)$ in serum and CSF against either crude extracts of juveniles and adults or metabolites of adult worms (Kamiya, 1975; Tharavanij, 1979; Chen, 1986).

The detection of antigen $(\mathrm{Ag})$ rather than $\mathrm{Ab}$ may have greater clinical use as has been exemplified in monkeys experimentally infected with $A$. cantonensis larvae (Chen, Suzuki \& Lin, 1973). The detection of antigens which circulate (cir- $\mathrm{Ag}$ ) has also achieved emphasis elsewhere; these molecules usually have weak antigenicity and unbalanced $\mathrm{Ag} / \mathrm{Ab}$ ratios (Smith, Karr, Lykins \& Ristic, 1972; Phillips \&
Draper, 1975). There were at least two kinds of cir-Ag (Yamashita, Saito, Sato, Takai, Watanabe \& Otsuru, 1979 ) in the blood of rates experimentally infected with $A$. cantonensis. However, the detection of cir-Ag was not easy and cross-reactivity occurred like that reported in Bancroftian filariasis (Prasad, Reddy \& Harinath, 1983). Hybridoma technology provides one way of increasing the specificity and reproducibility of the diagnostic test.

In this study, we analysed the components of L3 ES of $A$. cantonensis and evaluated their antigenicity. Two hybridoma cell lines were established and each $\mathrm{Mc} \mathrm{Ab}$ secreted recognized a single circulating ES molecule from the $\mathrm{L} 3$ but with different epitope specificities. We developed sensitive ELFA immunodiagnosis using combined monoclonal antibodies.

\section{MATERIALS AND METHODS}

Parasite antigens. A strain of Angiostrongylus cantonensis from Taiwan has been maintained for more than 10 years in our laboratory in adult Wistar rats and water snails, Biomphalaria glabratus. Third-stage larvae (L3) were harvested and collected (Chen, Tang $\&$ Lee, 1981) and more than 1000 were homogenized by sonication and extracted overnight at $4^{\circ} \mathrm{C}$ in a refrigerator. The extract was centrifuged ( 30 min, $11,000 \mathrm{~g}$ ) and the supernatant, 'somatic antigens', was 
assayed for protein (Bradford, 1976). These antigens were used as: (1) $\mathrm{Ag}$ to immunize $\mathrm{BALB} / \mathrm{c}$ mice to produce hybridoma cell lines; (2) in indirect ELISA to screen the hybridomas; and (3) to establish total L3 protein profiles for immunoblot analysis. Somatic preparations of Toxocara cati and Clonorchis sinensis were extracted by the same procedures as described above and stored at $-20^{\circ} \mathrm{C}$ until use. These last two species were collected from cats infected experimentally by another group in our laboratory.

Radiolabelled metabolic products. Intact and alive L3 were cultured at $37^{\circ} \mathrm{C}$ for $1-2$ days in $1-2 \mathrm{ml}$ of tissue culture medium minus methionine (Leibovitz's L 15, Gibco Ltd), containing penicillin $\left(100\right.$ i.u. $\left.\mathrm{ml}^{-1}\right)$, streptomycin $(0.1 \mathrm{mg}$ $\mathrm{ml}^{-1}$ ) and $3.7 \mathrm{mBq} \mathrm{ml}^{-1}$ of ${ }^{35} \mathrm{~S}$-methionine (specific activity $>43.03 \mathrm{TBq} \mathrm{mmol}^{-1}$, New England Nuclear). At the end of the incubation, radiolabelled proteins were recovered from culture medium by precipitation with ice-cold ethanol $(80 \%$, $v / v$, final concentration) (Parkhouse \& Clark, 1983). The total radioactivity present in the ES was estimated by assays on precipitates of aliquots with $10 \%(\mathrm{w} / \mathrm{v}) \mathrm{TCA}$ in a liquid scintillation counter (LKB 1217 RACK BETA).

Hybridomas. Cell fusion was effected between splenic lymphocytes from BALB/c mice immunized three times with total L 3 somatic antigens and myeloma cells from the FO cell line. The hybridomas were developed according to the procedures of Köhler \& Milstein (1975) using polyethylene glycol (Merck, mol.wt 1500). Antibody activity of culture supernatant was assessed by indirect ELISA using $A$. cantonensis $\mathrm{L} 3$ extract. Ascites containing $\mathrm{Mc} \mathrm{Ab}$ produced in pristane-primed mice were collected and stored at $-20^{\circ} \mathrm{C}$ until use.

Isotyping and purification of monoclonal antibodies. An isotyping kit (Southern Biotechnology Associates Inc., U.S.A.) was used to distinguish the classes and subclasses of the $\mathrm{Mc} \mathrm{Ab}$ secreted. Ascites containing Mc Ab of the IgG2a subclass were purified with a Protein A-Sepharose CL-6B immunosorbent column (Bio-Rad Laboratories, U.S.A.) (Goding, 1978). Eluted Ig were collected, neutralized to $\mathrm{pH}$ 7.0, desalted with a prepacked PD-10 column (Sephadex G25, bed volume $9 \mathrm{ml}$, Pharmacia Fine Chemicals $\mathbf{A B}$, Sweden), and then concentrated by lyophilization. The Mc $\mathrm{Ab}$ of IgM class was purified by gel filtration chromatography with a Sephacryl S 300 (Pharmacia) column. Fractions of the first absorbance peak eluted at $280 \mathrm{~nm}$ were collected, desalted and lyophilized as stated above.

Radio-immunoprecipitation. Soluble radioactive material was incubated with pooled sera $(5-15 \mu 1)$ either from rats infected with $A$. cantonensis or from patients with eosinophilic meningoencephalitis, respectively. Antigen-antibody complexes were precipitated with an excess of either goat anti-rat Ig or goat anti-human Ig antisera (Kirkegaard \& Perry Laboratories, kpl, U.S.A.), and washed as described by Parkhouse \& Clark (1983). Control precipitates were prepared from pooled normal uninfected rat and human sera. ${ }^{35} \mathrm{~S}$-Labelled precipitates were divided into two parts: one was counted for total radioactivity, and the other was resolubilized in PBS and analysed by SDS-polyacrylamide gel electrophoresis (SDS-PAGE).

Sera. Rat sera were obtained at intervals from Wistar rats infected orally with $80 \mathrm{~L} 3$ of $A$, cantonensis (see Yoshimura \& Soulsby, 1976) and stored at $-20^{\circ} \mathrm{C}$ until use. Human sera and CSF, obtained from patients with the clinical symptoms of eosinophilic meningoencephalitis, were collected mainly at the National Taiwan University Hospital. Control sera were collected from confirmed patients of schistosomiasis, toxocariasis, clonorchiasis and taeniasis.
SDS-PAGE. Samples were reduced (Parkhouse \& Clark, 1983) and electrophoresed on $10 \%$ gel slabs using Bio-Rad equipment. The gel slabs were fixed, dried and autoradiographed. Apparent mol.wt of $A$. cantonensis components were determined against standard marker proteins (Pharmacia, Sweden). Thereafter a mini-gel system with a $50 \mathrm{~mm}$ running gel and $20 \mathrm{~mm}$ stacking gel was used. Proteins electrophoresed on the mini-gel slabs were then transferred to nitrocellulose (NC) paper to analyse their antigenicity.

Protein blotting. The transfer technique of Gershoni \& Palade (1983) was modified slightly. The mini-gel slabs were equilibrated in Tris-glycine transfer buffer, overlaid with $\mathrm{NC}$ paper (Immobilon transfer membranes, Millipore, U.S.A.), sandwiched between filter papers in a gel holder, and the proteins transferred for $1 \mathrm{~h}$ at $0.5-1.0 \mathrm{~A}$ in a transfer tank (Hoefer scientific instruments, U.S.A.). The NC paper was washed overnight with $6 \mathrm{M}$-urea dissolved in PBS containing $0.05 \%$ Tween-20 (PBST) to recover electrophoresed proteins which had been transferred. The NC paper was rinsed in PBST and blocked in PBS containing 3\% skimmed milk for 1 $h$ at room temperature, rinsed twice in PBS, cut into strips, and exposed individually to each $\mathrm{Mc} A \mathrm{~b}$ in ascites at the 1:500 dilution level in PBS for $3 \mathrm{~h}$ at $37^{\circ} \mathrm{C}$. The treated strips were rinsed three times in PBST and exposed to horseradish peroxidase-labelled goat anti-mouse $\mathrm{Ig}$ antiserum (kpl) purified by affinity chromatography and diluted 1:1000 in PBS for $2 \mathrm{~h}$ at $37^{\circ} \mathrm{C}$. The reacted NC strips were developed in PBS containing $0.1 \mu \mathrm{g} \mathrm{ml}^{-1}$ 3,3'-diaminobenzidine (DAB, Sigma, U.S.A.) and $0.01 \% \mathrm{H}_{2} \mathrm{O}_{2}$.

ELISA additivity test. To distinguish whether or not $\mathrm{Mc}$ Ab recognized different antigen epitopes (Friguet, DjavadiOhaniance, Pages, Bussard \& Goldberg, 1983), a basic ELISA test (Voller, Bidwell \& Bartlett, 1979) was performed using horseradish peroxidase as the enzyme tracer. The saturation curves of the $\mathrm{Ag}$ by each $\mathrm{Mc} \mathrm{Ab}$ were determined by the indirect ELISA, then the epitope specificity of the Mc $\mathrm{Ab}$ was analysed by adding two ascitic fluids to each well in amounts determined to be sufficient for each to saturate the coated Ag. The amount of bound $\mathrm{Ab}$ was assayed and the additivity index (A.I.) was calculated following the equation proposed by Friguet et al. (1983).

ELFA. The ELISA procedure was adapted to ELFA (Yolken \& Stopa, 1979) by substituting a fluorogenic substrate for the color-producing substrate. Optimal dilutions of reagents were determined by checkerboard titration. A double-Ab sandwich method was used to detect cir-Ag in sera from immune rats, patients and in CSF. An aliquot of $0.010 \mu \mathrm{g}$ ascitic protein of TD2 was coated per well on the MicroFLUOR ' $B$ ' plate (Dynatech Laboratories Inc., U.S.A.). The plates were incubated overnight at $4^{\circ} \mathrm{C}$, washed four times with PBST using a 96-well plate washer (Dynateck), and $50-\mu 1$ samples of either a clinical or an experimental specimen were added to the wells and incubated for $3 \mathrm{~h}$ at $37^{\circ} \mathrm{C}$ and overnight at $4^{\circ} \mathrm{C}$. The plates were washed and $0.015 \mu \mathrm{g}$ of ascitic protein of $3 \mathrm{~A} 5$ was added to each well and incubated for $1 \mathrm{~h}$ at $37^{\circ} \mathrm{C}$. The plates were washed, and affinity-purified phosphatase-labelled goat anti-mouse Ig heavy chain $\mu$ antiserum (human serum absorbed, kpl) diluted 1000 -fold was added. The plates were incubated for 1 $h$ at $37^{\circ} \mathrm{C}$, washed and a $100-\mu 1$ volume of 4 -methylumbelliferyl phosphate (MUP, Sigma) diluted to $10^{-4} \mathrm{M}$ in $0.03 \mathrm{M}$ diethanolamine buffer ( $\mathrm{pH} 9.8$ ) containing $10^{-5} \mathrm{M}-\mathrm{MgCl}_{2}$ $6 \mathrm{H}_{2} \mathrm{O}$ was added to each well and incubated for $30 \mathrm{~min}$ at $37^{\circ} \mathrm{C}$. The amount of fluorescence in each well was measured directly in a MicroFLUOR reader (Dynatech) and expressed as F.U. A background reading was taken from the well 
containing the substrate alone and the value subtracted from those of the specimens, either normal rat or human sera were run with each test as negative controls.

\section{RESULTS}

Antigenicity of excretory-secretory antigens from third-stage larvae

The components detected in the sccretions of Angiostrongylus cantonensis L3 are highly antigenic to infected hosts. The immunoprecipitates of sera from cither infected rats or patients showed significantly higher values than did sera from the normal controls (Table 1). Further analysis by resolubilization of immunoprecipitates of ES Ag with SDS PAGE (Fig. 1) illustrated that only molecules of mol.wts 79,000 , 65,000 and 43,000 were precipitated by pooled sera from infected rats. One major band of mol.wt 91,000 did not appear in the autoradiograph of electrophoresed immunoprecipitates (Fig. 1, lane 2).

TABle 1-Antigenicity of A. cantonensis L3 ES

\begin{tabular}{lc}
\hline Serum from & $\begin{array}{c}\text { Immunoprecipitation } \\
\text { (\% of TCA precipitation) }\end{array}$ \\
\hline Normal human & $16.6 \pm 5.6$ \\
Angiostrongyliasis patient & $53.3 \pm 8.4$ \\
Normal rat & $13.5 \pm 3.7$ \\
Infected rat & $29.4 \pm 6.6$ \\
\hline
\end{tabular}

\section{Hybridoma production and characterization}

Six hybridoma cell lines secreting $\mathrm{Ab}$ against either somatic or ES Ag from L3 were established. Two Mc $\mathrm{Ab}$ recognized a molecule of mol.wt 91,000 as shown by immunoblot (Fig. 2) and radio-immunoprecipitation (Fig. 3). The antibodies are of IgG2a subclass (TD2) and of IgM class (3A5). The A.I. for TD2 and

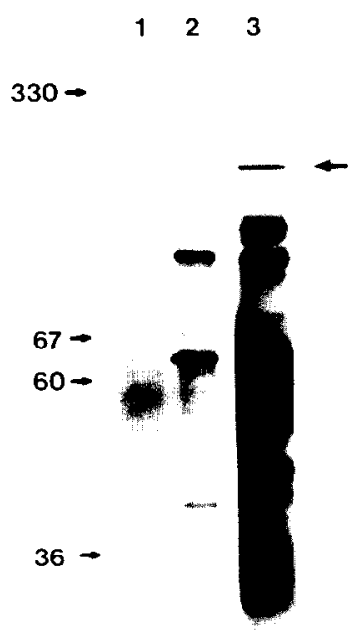

Fig. 1. Autoradiograph of labelled L3 excretory-secretory products (ES) co-precipitated by pooled normal human sera (1) and pooled sera from patients (2) with eosinophilic meningocncephalitis. Protcin profilc of L3 ES paralleled (3). Position of mol.wt standards shown on the left. Arrow at right indicates position of molecules mol.wt 91,000 .

3A5 was $83.0 \%$ (Table 2) which revealed that the two $\mathrm{Mc} \mathrm{Ab}$ showed additive binding and that the $\mathrm{Mc} \mathrm{Ab}$ from TD2 and 3A5 cell lines have different epitope specificities within the mol.wt 91,000 molecule of L3 ES.

Detection of circulating antigens of mol.wt 91,000 in sera from infected rat

Circulating Ag of mol.wt 91,000 was detected by a
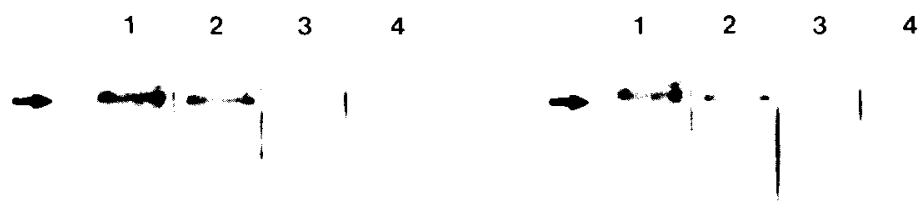

B

FIG. 2. Immunoblots of worm somatic extracts and excretory secretory products (ES). Protein blots of somatic extract of Angiostrongylus cantonensis L3 (1), of Toxocara cati adult (3), of Clonorchis sinensis adult (4) and L3 ES of $A$. cantonensis (2) were probed with monoclonal antibodies (Mc Ab) TD2 (A) and 3A5 (B). Molecules of mol.wt 91,000 were recognized by both Mc Ab (arrows). 
TABLe 2-The ELISA additivity TEST WITH MONOClONAL ANTIBODIES AGAINSI $A$. cantonensis L3

\begin{tabular}{lccc}
\hline $\begin{array}{l}\text { Monoclonal } \\
\text { antibodies }\end{array}$ & $\begin{array}{c}\text { Optical density } \\
\text { at } 492 \mathrm{~nm}\end{array}$ & $\begin{array}{c}\text { Theoretical } \\
\text { sum }\end{array}$ & $\begin{array}{c}\text { Additivity } \\
\text { index }(\%)\end{array}$ \\
\hline TD2 $(1: 80)$ & 0.712 & - & - \\
3A5 $(1: 160)$ & 0.873 & - & - \\
TD2 + 3A5 & 1.450 & 1.585 & 83.0 \\
TD2 + TD2 & 0.771 & 1.424 & 8.3 \\
3A5 + 3A5 & 0.887 & 1.746 & 1.6 \\
\hline
\end{tabular}

double-Ab sandwich ELFA in sera from infected rats collected from 8 to 180 days after infection (Fig. 4). Similar periodicity appeared in two circumstances: when the plate was coated with TD2 and the test sera were screened by $3 \mathrm{~A} 5$ and phosphatase-labelled antimouse $\mu$ chain conjugate; and alternately when the plate was coated with $3 \mathrm{~A} 5$ and screened by TD2 and phosphatase-labelled anti-mouse $\gamma$ chain conjugate. Two F.U. peaks of reactivity were recorded for sera taken from rats 18 and 44 days after infection (Fig. 4).

\section{Immunodiagnosis of human angiostrongyliasis}

Qualitative and quantitative assays for the mol.wt 91,000 molecules in sera and CSF of 35 patients with eosinophilic meningoencephalitis were done by double$\mathrm{Ab}$ sandwich ELFA against TD2. The negative/ positive cutoff was calculated as the mean F.U. from six normal human sera run with the test plus 2 s.D. values. All CSF and most sera $(88 \%, 31 / 35)$ were positive (Fig. 5). The average F.U. of CSF ( $837 \pm 95)$
Fig. 3. Autoradiograph of labelled L3 somatic components (1 and 3) and L3 excretory-secretory products (ES) (2 and 4) co-precipitated by monoclonal antibodies (Mc Ab) TD2 (1 and 2) and 3A5 (3 and 4). Molecules of mol.wt 91,000 are indicated by an arrow.

was greater than that for sera $(627 \pm 71)$. Cross reactivity was tested on five pools of infected sera collected from confirmed patients of schistosomiasis, toxocariasis, clonorchiasis and taeniasis. Cross-reactions were significantly lower than the cutoff value calculated from reactions between normal human serum in the test system.

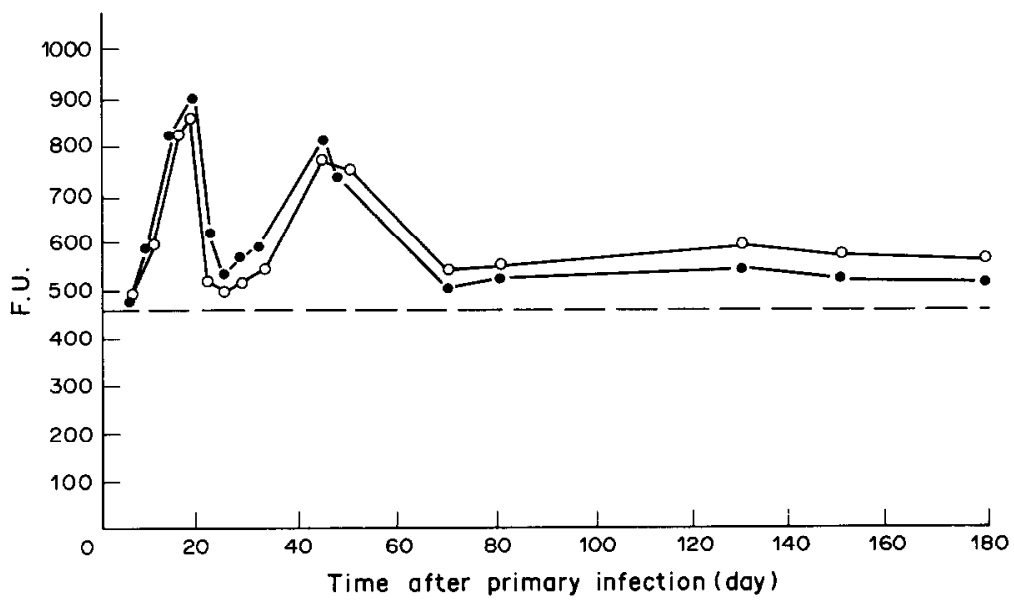

FIG. 4. Double antibody sandwich ELFA detecting circulating antigen of mol.wt 91,000 in infected rat sera. Rats infected with 80 Angiostrongylus cantonensis L3. MicroFLUOR 'B' plate coated first with $0.010 \mu \mathrm{g}$ ascitic protein from monoclonal antibody (Mc Ab) TD2/ well, and screened by $\mathrm{Mc}$ Ab $3 \mathrm{~A} 5$ and phosphatase-labelled anti-mouse $\mu$ chain antiserum (O). Plate coated first with $0.015 \mu \mathrm{g}$ ascitic protein from $3 \mathrm{~A} 5$, and then screened by $\mathrm{Mc} \mathrm{Ab}$ TD2 and phosphatase-labelled anti-mouse chain $\gamma$ antiserum $(O)$. A value exceeding 467 fluorescence unit (F.U.) (--), equal to the average counts from normal rat sera plus 3 S.D., was considered to be positive. 


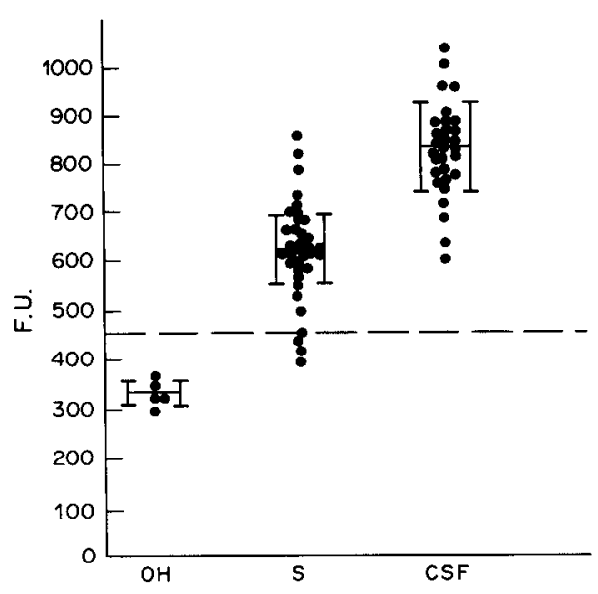

FIG. 5. Double antibody sandwich ELFA detecting circulating antigens of mol.wt 91,000 in sera (S) and cerebrospinal fluids (CSF) of patients with eosinophilic meningoencephalitis. Inter-helminth cross reactivity examined on five pools of sera from other helminth-infected $(\mathrm{OH})$ patients with schistosomiasis, toxocariasis, clonorchiasis and taeniasis. A value exceeding 370 fluorescence unit (F.U.) (---), equal to the average counts from normal human sera plus 3 S.D., was considered to be positive.

\section{DISCUSSION}

The level of circulating $\mathrm{Ab}$ detected either in the serum or CSF of patients with $A$. cantonensis may not be clearly diagnostic of infection because of the delay in the appearance, persistence in the circulation, and the high level of cross-reactivity of the $\mathbf{A b}$ (des Moutis, Ouaissi, Grzych, Yarzabal, Haque \& Capron, 1983). We therefore sought to detect cir-Ag. Indeed, such $\mathrm{Ag}$ is present in the circulation before specific $A b$ appears and unlike $A b$ does not persist for long in the circulation after the parasite is lost. Circulating $\mathrm{Ag}$ from $A$. cantonensis was first recovered in the sera of experimentally infected rats by Yamashita et al. (1979). They demonstrated $\mathrm{Ag}$ in sera of $33.3 \%$ of cyclophosphamide-treated and $20.0 \%$ of untreated rats infected with $100 \mathrm{~A}$. cantonensis larvae and proposed that the detection of cir- $\mathrm{Ag}$ was a highly sensitive method for diagnosis. Our results support this proposition.

The L3 ES of $A$. cantonensis is composed of many complex molecules (Fig. 1, lane 3) as traced by radioisotope $-{ }^{35} \mathrm{~S}$. More than half of the total radioactivity appeared in immunoprecipitates from reactions with sera from patients with eosinophilic meningoencephalitis (Table 1). The electrophoresis patterns of resolubilized immunoprecipitates indicate that many molecules, including some major ones, were not precipitated (Fig. 1, lane 2). That is, the molecules show weak antigenicity in both humans and rats.

Two $\mathrm{Mc} \mathrm{Ab}$ which recognize weak antigenic molecules of mol.wt 91,000 were found by chance when screening hybridoma secretions. Figure 3 shows that both TD2 and 3A5 can catch the mol.wt of 91,000 molecules among radiolabelled ES and somatic antigens. These two Mc Ab belonged to different Ig classes and showed different epitope specificities. Observations on combinations of reactions between the $\mathrm{Mc} \mathrm{Ab}$ and the $A$. cantonensis antigens established ELFA as a sensitive immunoassay for angiostrongyliasis. The system was tested firstly against sera from experimentally infected rats either with TD2 or $3 A 5$ as the primary reaction antibody to detect molecules with a mol.wt of 91,000 in the circulation of the infected rats. Two peaks of $\mathrm{Ag}$ activity at 18 and 44 days after infection (Fig. 4) indicated that the cir-Ag of mol.wt 91,000 is common between stages of $A$. cantonensis (data not shown). The antigen is secreted into the rat circulation largely when the worms migrated from rat brain to the pulmonary arteries. In human cases, F.U. obtained from sera from most patients and all CSF are significantly higher than those from normal sera; the average value for the CSF was greater than that for sera. The difference between sera and CSF may result because $\mathrm{L} 3$ of $A$. cantonensis do not usually develop into the adult stage in humans and are always retained in the central nervous system (CNS), so that the amount of ES materials is greater in CSF than in the serum. Alternatively it may arise because the protein content of the CSF is only about $0.5 \%$ of that of serum (Hochwald, 1970), although $\gamma$-globulin within the CNS which was synthesized locally has been reported in patients with a variety of neurological diseases (Lippincott, Korman, Lax \& Corcoran, 1965). The cirAg of mol.wt 91,000 which traversed the brain-blood barrier and appeared in human circulation may combine with circulating $\mathrm{Ab}$ to be eliminated as an immune complex. Molecules which remain in the CSF may persist longer at increasing concentrations as the worms continue to secrete and excrete them into the brain.

Application of $\mathrm{Mc} \mathrm{Ab}$ in immunodiagnosis is recommended by Felice \& Siracusano (1987). Mc Ab offer several advantages including: decreased falsepositive reactions; decreased cross reactivity (because each $\mathrm{Mc} \mathrm{Ab}$ usually recognizes only one antigen determinant unique to the parasite of interest); and increased reproducibility and standardization of the test. However sensitivity in diagnosis may be lost because of the single epitope specificity of $\mathrm{Mc} \mathrm{Ab}$. This problem was overcome here by using two $\mathrm{Mc} A \mathrm{~B}$ which react with different epitopes on the same major cir-Ag. Sensitivity was increased remarkably by substituting a fluorogenic for the colorigenic substrate usually used in ELISA. ELFA required about 100 -fold less enzyme than did ELISA (Yolken \& Stopa, 1979).

Some problems within the present immunodiagnostic system remain to be resolved. The sources of the cir-Ag have not yet been confirmed, but we are sure that they are not shed from the L3 cuticle because the surface of the $\mathrm{L} 3$ did not react in an indirect fluorescent Ab test with TD2 (data not shown). Secondly, although both of these two Mc Ab could not react with 
somatic extracts of either Toxocara canis or Clonorchis sinensis (Fig. 2), the sample size of cross reactivity is still too small and only species from the classes Trematoda and Cestoda were studied, and only one serum pool from another nematode infection was used. Finally, ES molecules were more abundant in human CSF than in serum and CSF is therefore a better test material than serum for immunodiagnosis, but spinal puncture is a cumbersome technique which requires educated skill. The assay could be done however whenever patients with neurological symptoms are tapped for CSF for other tests. The ELFA on CSF nevertheless is not as convenient to do as other assays for cir-Ag on blood and urine developed so far, e.g. for Trypanosoma lewisi by D'Alesandro (1972); Plasmodium species by Smith et al. (1972); Onchocera volvulus by des Moutis et al. (1983); Wuchereria bancrofti by Franks (1946) and Prasad et al. (1983); Schistosoma mansoni by Gold, Rosen \& Weller (1969), Deelder, Klappc, van den Aardweg \& van Meerbeke (1976) and Bout, Santoro, Carlier, Bina \& Capron (1977); and for S. japonicum by Hirata \& Akusawa (1975) and Hirata (1976).

\section{REFERENCES}

Alicata J. E. \& JindRaK K. 1970. Angiostrongyliasis in the Pacific and Southeast Asia. In: American Lectures in Tropical Medicine (Edited by Thomas C. C.). Springfield, Illinois.

Bout D., Santoro F., Carlier Y., Bina J. C. \& Capron A. 1977. Circulating immune complexes in schistosomiasis. Immunology 33: 17-22.

BradFord M. M. 1976. A rapid and sensitive method for the quantitation of microgram quantities of protein utilizing the principle of protein-dye binding. Analytical Biochemistry 72: 248-254.

Chen S. N., Suzukı T. \& LiN K. H. 1973. Studies on immunodiagnosis of angiostrongyliasis. 1. Detection of antigen and antibody in serum and cerebrospinal fluid. Journal of Formosan Medical Association 72: 161-166.

Chen S. N., Tang T. \& Lee S. J. 1981. The in vitro cultivation of the first and third stage larvae and adult worm of Angiostrongylus cantonensis. Proceedings of the National Science Council, Republic of China, Part B 5: 375-384.

Chen S. N. 1986. Enzyme-linked immunosorbent assay (ELISA) for the detection of antibodies to Angiostrongyius cantonensis. Transactions of the Royal Society of Tropical Medicine and Hygiene 80: 398-405.

Cross J. H. 1978. Clinical manifestation and laboratory diagnosis of eosinophilic meningitis syndrome associated with angiostrongyliasis. Southeast Asian Journal of Tropical Medicine and Public Health 9: 161-170.

D'Alesandro P. A. 1972. Trypanosoma lewisi: production of exoantigens during infection in the rat. Experimental Parasitology 32: 149-164.

Deelder A. M., Klappe H. T. M., Aardweg G. J. M. J. van Den \& Meerbeke E. H. E. M. van 1976. Schistosoma mansoni: demonstration of two circulating antigens in infected hamsters. Experimental Parasitology 40: 189-197.

Felice G. K. \& Siracusano A. 1987. Monoclonal antibodies for immunodiagnosis of human hydatidosis. Parasitology Today 3: 25-26.

Franks M. B. 1946. Specific soluble antigen in the blood of filarial patients. Journal of Parasitology 32: 400-406.

Friguet B., Duavadi-Ohaniance L., Pages J., Bussari A. \& Goldberg M. 1983. A convenient enzyme-linked immunosorbent assay for testing whether monoclonal antibodies recognize the same antigenic site. Application to hybridomas specific for the $\beta_{2}$-subunit of Escherichia coli tryptophan synthase. Journal of Immunological Methods 60: 351358 .

Gershoni J. M. \& Palade G. E. 1983. Protein blotting: principles and applications. Analytical Biochemistry 131: $1-15$.

Goding J. W. 1978. Use of staphylococcal protein A as all immunological reagent. Journal of Immunological Methods 20: 241-254.

Gold R., Rosen F. S. \& Weller T. H. 1969. A specific circulating antigen in hamsters infected with Schistosoma mansoni. Detection of antigen in serum and urine, and correlation between antigenic concentration and worm burden. American Journal of Tropical Medicine and Hygiene 18: 545-551.

Hirata M. \& Akusawa M. 1975. Circulating antigen in animals infected with Schistosoma japonicum. 1. Detection and characteristics of circulating antigen in infected rabbits. Japanese Journal of Parasitology 24: 250-254.

Hirata M. 1976. Circulating antigen in animals infected with Schistosoma japonicum. 2. Appearance of circulating antigen in infected mice. Japanese Journal of Parasitology 25: 396-401.

Hochwald G. M. 1970. Influx of serum proteins and their concentration in spinal fluid along the neuraxis. Journal of the Neurological Sciences 10: 269-278.

Kamiya M. 1975. Immunodiagnosis of Angiostrongylus cantonensis infection. In: Diagnostic Methods for Important Helminthiasis \& Amoebiasis in Southeast Asia \& the Far East (Edited by Harinasuta C. \& Reynolds D. C.), pp. 140-163. A Publication of the Central Coordination Board, SEAMEO-TROPMED Project, Bangkok, Thailand.

KOHLER G. \& MiLSTEIN C. 1975. Continuous cultures of fused cells secreting antibody of predefined specificity. Nature (London) 256: 495-497.

Lippincott S. W., Korman S., Lax L. C. \& Corcoran C. 1965. Transfer rates of $\gamma$-globulin between cerebrospinal fluid and blood plasma (results obtained on a series of multiple sclerosis patients). Journal of Nuclear Medicine 6 : 632-644.

Moutis I. des, Ouaissi A., Grzych J. M., Yarzabal L., HAQUe A. \& CAPRon A. 1983. Onchocerca volvulus: detection of circulating antigen of monoclonal antibodies in human onchocerciasis. American Journal of Tropical Medicine and Hygiene 32: 533-542.

Parkhouse R. M. E. \& Clark N. W. T. 1983. Stage specific secreted and somatic antigens of Trichinella spiralis. Molecular and Biochemical Parasitology 9: 319-327.

Phillips T. M. \& Draper C. C. 1975. Circulating immune complexes in schistosomiasis due to Schistosoma mansoni. British Medical Journal 2: 476-477.

Prasad G. B. K. S., Reddy M. V. R. \& Harinath B. C. 1983. Detection of filarial antigen in immune complexes in Bancroftian filariasis by ELISA. Indian Journal of Medical Research 78: 780-783.

SMith A. R., KarR L. J., Lykins J. D. \& Ristic M. 1972. Serum-soluble antigens of malaria: a review. Experimental Parasitology 31: 120-125.

Tharavanu S. 1979. Immunology of angiostrongyliasis. In: Studies on Angiostrongyliasis in Eastern Asia \& Australia (Edited by Cross J. H.), pp. 151-164. Special Publication of 
the U.S. Naval Medical Research Unit, No. 2, Taipei, Taiwan.

Voller A., Bidwell D. E. \& Bartlett A. 1979. Setting up ELISA. In: The Enzyme Linked Immunosorbent Assay (ELISA). A Guide with Abstracts of Microplate Applications (Edited by Voller A., Bidwell D. E. \& Bartlett A.), pp. 35-40. Dynatech Europe, Borough House, Rue du Pre, Guernsey, G.B.

Yamashita T., Saito Y., Sato Y., Takal A., Watanabe H. \& OTsuru M. 1979. Circulating antigens and immune com- plexes in the serum of rats infected with Angiostrongylus cantonensis. Japanese Journal of Parasitology 28: 393-401.

Yolken R. H. \& Stopa P. J. 1979. Enzyme-linked fluorescence assay: ultrasensitive solid-phase assay for detection of human rotavirus. Journal of Clinical Microbiology September: 317-321.

Yoshimura K. \& Soulsay E. J. L. 1976. Angiostrongylus cantonensis: lymphoid cell responsiveness and antibody production in rats. American Journal of Tropical Medicine and Hygiene 25: 99-107. 